\title{
Comportamento de cultivares de cafeeiros C. Arabica L. enxertados sobre cultivar 'Apoatã IAC 2258' (Coffea canephora)
}

\author{
Performance of C. Arabica L. Coffee cultivars grafted on 'Apoatã IAC 2258' cultivar \\ (Coffea canephora)
}

\section{Renato Fonseca de PaivaI Antônio Nazareno Guimarães Mendes ${ }^{I}$ Gladyston Rodrigues Carvalho"I Juliana Costa de Rezende ${ }^{\mathrm{I}}{ }^{*}$ Andre Dominghetti Ferreira ${ }^{\mathrm{II}}$ Alex Mendonça de Carvalho ${ }^{\mathrm{I}}$}

\section{RESUMO}

Objetivou-se no trabalho avaliar a influência da enxertia e do porta-enxerto 'Apoatã IAC 2258' no comportamento agronômico de sete cultivares de cafeeiro da espécie Coffea arabica cultivadas em solo isento de fitonematoides. $O$ ensaio foi conduzido em delineamento experimental de blocos casualizados em esquema fatorial ( $7 \mathrm{x}$ 3) com quatro repetições, sendo sete cultivares de Coffea arabica ('Obatã IAC 1669-20', 'Acauã', 'Oeiras MG 6851', 'Catucaí Amarelo 2SL', 'Topázio MG 1190', 'IBC Palma II’ e 'Paraíso MG H 419-1') e três tipos de enxertia (muda enxertada, auto enxertada e pé franco). Foi avaliada a produtividade média de quatro anos, uniformidade de maturação, percentagem de frutos chochos e classificação do grão por tipo de peneira. As cultivares enxertadas estudadas apresentaram comportamento agronômico satisfatório em todas as características avaliadas e semelhante ao pé-franco. Em áreas isentas de fitonematoides, à exceção das cultivares 'IBC Palma II' e 'Paraíso MG H 419-1', as demais cultivares estudadas são adequadas opções de escolha para uso como copas. A técnica da enxertia com o uso do porta-enxerto 'Apoatã IAC 2258' em área isenta de fitonematoides não se justifica para incremento de produtividade.

Palavras-chave: café, tipos de enxertia, produtividade, cultivares.

\section{ABSTRACT}

This research had the objective to evaluate the effect of grafting and 'Apoatã IAC 2258' cv. as rootstock on agronomic performance of seven Coffea arabica cvs. planted in area nematodes free. The experimental design was randomized blocks using a $7 \times 3$ factorial scheme with four repetitions. The treatments were 'Obatã IAC 1669-20', 'Acauã', 'Oeiras MG 6851', 'Catucaí Amarelo 2SL', 'Topázio MG 1190',
'IBC Palma II' and 'Paraíso MG H 419-1' coffee cultivars grafted on 'Apoatã IAC 2258', self grafted (meaning a cultivar was grafted on a rootstock of the same cultivar) and no grafting. The characteristic evaluated was the average yield of beans between the first and the fourth year, the fruit maturation stage, floating grain percentage and the bean size. The grafting cultivars showed good agronomic performance in the characteristic evaluated. The performance in coffee cultivars grafted on 'Apoatã IAC 2258' is the same in no grafting. In nematodes free areas, with the exception of the cultivars 'IBC Palma II' and 'Paraíso MG H 419-1', all cultivars are good choices to be used as scions. The grafting technique using 'Apoatã IAC 2258' as rootstock on area free of nematodes is not justified for yield increase.

Key words: coffee, grafting, yield, cultivars.

\section{INTRODUÇÃO}

A utilização de mudas enxertadas em porta enxertos tolerantes à fitonematoides é uma das alternativas que viabiliza o cultivo do cafeeiro em áreas infestadas, uma vez que o controle químico é oneroso, resultando em contaminação ao ambiente e risco toxicológico para o homem. Dessa forma, tem sido utilizado o sistema radicular de $\boldsymbol{C}$. canephora para controlar os danos causados pelos nematoides em cultivares de C. arabica (FAHL et al., 1998).

Além da resistência de determinados portaenxertos aos fitonematoides, alguns autores destacam um maior desenvolvimento do sistema radicular e da

IDepartamento de Agricultura (DAG), Universidade Federal de Lavras (UFLA), Lavras, MG, Brasil.

IIUnidade Regional do Sul de Minas (URESM), Empresa de Pesquisa Agropecuária de Minas Gerais (EPAMIG), 37200-000, Lavras, MG, Brasil. E-mail: julianacosta@epamig.br. *Autor para correspondência. 
parte aérea e uma maior eficiência na absorção de nutrientes em relação às plantas não enxertadas, podendo essas plantas serem utilizadas em áreas isentas de nematoides, aumentando a produtividade da lavoura (FERREIRA et al., 2010).

Se por um lado o Coffea arabica é mais susceptível a nematoides do que o Coffea canephora, por outro lado, trata-se de um café de qualidade de bebida muito superior. O efeito da enxertia pode variar com o material genético utilizado nas diversas combinações enxerto/porta-enxerto. Em adição, parece existir uma interação fisiológica entre a parte aérea e o sistema radicular, a qual proporciona melhor desempenho às plantas enxertadas, mesmo em condições isentas de nematoides (ALFONSI et al., 2005). Dessa forma, conhecer os efeitos e as possibilidades do processo de enxertia, no qual o portaenxerto possa aumentar a adaptabilidade da planta enxertada, é de grande relevância para a cafeicultura.

Muitos trabalhos têm sido realizados com o objetivo de estudar o comportamento agronômico em relação à produtividade e características dos frutos de cultivares de café, mas, com relação à enxertia, as pesquisas em condições de campo ainda são bastante escassas. Dessa forma, objetivou-se no presente trabalho avaliar a influência do uso da técnica da enxertia e do porta-enxerto 'Apoatã IAC 2258' na produtividade e características de frutos de sete cultivares da espécie Coffea arabica $L$., visando a conhecer a viabilidade da utilização da enxertia em condições isentas de nematoides.

\section{MATERIAL E MÉTODOS}

O experimento foi instalado em janeiro de 2004 em área isenta de fitonematoides do Setor de Cafeicultura da Universidade Federal de Lavras (UFLA), Lavras-MG. Utilizou-se o esquema fatorial 7x3, com sete cultivares de Coffea arabica ('Obatã IAC 1669-20’, ‘Acauã', ‘Oeiras MG 6851’, ‘Catucaí Amarelo 2SL', ‘Topázio MG 1190’, 'IBC Palma II’e ‘Paraíso MG H 419-1') e três tipos de enxertia (muda enxertada, autoenxertada e pé franco), com quatro repetições. A utilização da auto-enxertia (enxertia de uma cultivar sobre ela mesma) teve por objetivo isolar o efeito do processo de enxertia. $O$ processo de enxertia utilizado foi do tipo garfagem hipocotiledonar.

O experimento foi implantado em blocos casualizados, com quatro repetições e espaçamento de 3,00x0,60m. As parcelas foram constituídas por linhas de sete plantas, considerando-se como úteis as cinco plantas centrais. Foram utilizados os tratos culturais normalmente empregados para a cultura do cafeeiro na região. As avaliações foram realizadas a partir dos 30 meses, compreendendo as seguintes características: produtividade de café beneficiado em sacas de $60 \mathrm{~kg} \mathrm{ha}^{-1}$ de quatro colheitas (de 2007 a 2010), uniformidade de maturação, porcentagem de frutos chochos e classificação por peneira das colheitas 2008, 2009 e 2010.

A produção foi medida imediatamente após a colheita em litros de “café da roça” por parcela, sendo a colheita realizada entre os meses de maio a agosto de cada ano. Posteriormente, foi realizada a conversão para sacas de $60 \mathrm{~kg}$ de café beneficiado.ha-1 ${ }^{-1}$ por meio do rendimento de uma amostra de três litros do café coletada por ocasião da colheita. O estádio de maturação dos frutos foi avaliado calculando-se a porcentagem de frutos verdes, cereja e passa/secos, por meio de contagem em amostras de 300 frutos por parcela.

A porcentagem de frutos chochos foi avaliada utilizando-se a metodologia proposta por ANTUNES FILHO \& CARVALHO (1954), em que 100 frutos maduros são colocados em água e se consideram chochos aqueles que permanecem na superfície. Utilizaram-se 300 gramas de café beneficiado de cada repetição para classificação em peneira, sendo considerados apenas os grãos retidos nas peneiras 19, 18 e 17 (BRASIL, 2003).

Os dados foram analisados utilizando-se o aplicativo computacional Sisvar (FERREIRA, 2008), adotando-se significância de 5\% de probabilidade, para o teste F. A análise estatística da produtividade foi realizada em esquema de parcelas subdivididas no tempo, acrescentando-se assim mais um fator na análise, resultando em uma comparação em fatorial triplo para esta variável (cultivares, tipos de enxertia e colheita). A partir da detecção de diferenças significativas entre tratamentos e suas interações, foram feitos os desdobramentos e as médias de todas as características avaliadas foram comparadas entre si pelo teste de Scott Knott.

\section{RESULTADOS E DISCUSSÃO}

De acordo com a análise de variância conjunta para a característica produtividade, houve efeito significativo em nível indicado pelo teste F para todas as interações duplas possíveis entre os fatores estudados, assim como para a interação tripla cultivares $\mathrm{x}$ tipos de enxertia $\mathrm{x}$ colheita (Tabela 1 ), refletindo diferentes sensibilidades das cultivares aos tipos de enxertia nas colheitas estudadas.

Nota-se que as cultivares Acauã, Obatã e Paraíso tiveram suas produtividades iniciais 
Tabela 1 - Resumo da análise de variância e coeficientes de variação da análise conjunta e subdividida no tempo para produtividade em sacas de $60 \mathrm{~kg} \mathrm{ha}^{-1}$ de quatro colheitas (de 2007 a 2010), em função de diferentes cultivares e tipos de enxertia.

\begin{tabular}{lll}
\hline FV & GL & $\begin{array}{c}\text { Quadrados } \\
\text { Médios }\end{array}$ \\
\hline Bloco & 3 & $655,6^{*}$ \\
Cultivar & 6 & $2288,7^{*}$ \\
Tipo de enxertia & 2 & 603,7 \\
Cultivar*Tipo de enxertia & 12 & 341,4 \\
Erro 1 & 18 & 174,6 \\
Colheita & 3 & $116783,7^{*}$ \\
Erro 2 & 9 & 468,2 \\
Tipo de Enxertia* Colheita & 6 & $4200,5^{*}$ \\
Cultivar* Colheita & 18 & $1190,9^{*}$ \\
Cultivar*Tipo de enxertia*Colheita & 36 & $311,8^{*}$ \\
Erro 3 & 222 & 205,2 \\
Total & 335 & \\
CV 1 (\%) & & 25,3 \\
CV 2 (\%) & & 41,5 \\
CV 3 (\%) & & 27,4 \\
Média geral & & 52,1 \\
\hline
\end{tabular}

* Significativo em nível de 5\% de probabilidade pelo teste de F.

influenciadas negativamente pela enxertia e auto enxertia. Entretanto, na colheita seguinte, houve uma inversão nos resultados, na qual todas as cultivares enxertadas apresentaram produtividades iguais ou superiores às plantas auto enxertadas e pé franco. Independente da cultivar avaliada, as mudas enxertadas apresentaram produtividade superior sobre as demais na segunda e quarta colheitas.

Na cultura do café, um fator significativo que interfere na variação de sua produção, próprio de sua natureza fisiológica, é a alternância bienal, de altas e baixas produtividades. Esse fator é comumente atribuído à diminuição das reservas das plantas em anos de safra com altas produtividades, o que faz com que, em virtude do menor crescimento dos ramos plagiotrópicos, a produção no ano seguinte seja baixa (PEREIRA et al., 2011).

Com base nas médias das quatro colheitas, conclui-se que os resultados observados para produtividade estão de acordo com os obtidos em outros estudos destas cultivares (DIAS et al., 2005; PAIVA et al., 2010), evidenciando que, apesar das particularidades de cada colheita e da bienalidade marcante na cultura do café, os diferentes tipos de enxertia apresentam uma tendência de igualarem suas produtividades após dois biênios. Vale ressaltar que o ciclo de avaliação utilizado neste estudo foi suficiente para discriminar, com eficiência, o potencial produtivo das cultivares, visto que se trata de uma cultura perene e com quatro colheitas infere-se que a produção alcançada já é representativa (CARVALHO, 1989; FAZUOLI, 1977).

Analisando a média das quatro colheitas, observa-se que apenas a cultivar Paraíso demonstrou incompatibilidade com a enxertia (Tabela 2), corroborando resultados de DIAS et al. (2009). Esses autores, ao avaliarem a produtividade das mesmas cultivares utilizadas no presente trabalho, observaram inferioridade da produtividade da cultivar ‘Paraíso’ em relação às demais. Por outro lado, a cultivar 'IBC Palma II' enxertada e auto-enxertada apresentou produtividade superior às suas respectivas plantas de mudas pé franco. Sendo assim, esta cultivar foi influenciada positivamente pelo processo de enxertia e pela presença do porta enxerto. Esses resultados se deram em função das variações genéticas das cultivares, verificada também por outros autores (TOMAZ et al., 2005; 2008, CABOS et al., 2010).

Os resultados encontrados no presente trabalho evidenciam que a enxertia não traz benefícios em aumento de produtividade, podendo-se inferir que o uso da enxertia em cafeeiros em áreas isentas de fitonematoides é dispensável, pois, além de não proporcionar incremento em produtividade, sua utilização pode ser onerosa devido ao custo das mudas enxertadas ser, de maneira geral, o dobro do custo das mudas pé franco. Além disso, existe a dificuldade em se obter quantidades de mudas enxertadas disponíveis para utilização em lavouras comerciais.

Da mesma forma, BERTRAND et al. (2001) avaliaram, na Costa Rica, o desempenho de duas cultivares de C. arabica ('Caturra' e 'Catimor T5175') enxertadas em quatro variedades: Var Robusta (T3561 e T3757) de $\boldsymbol{C}$. canephora, var de liberica de $\boldsymbol{C}$. liberica (Hiern) e var. dewevrei (Lebrun) e observaram a redução de produtividade entre $10 \%$ a $48 \%$ quando comparado as duas cultivares de $\boldsymbol{C}$. arabica pé franco, confirmando a não viabilidade de utilização de mudas enxertadas.

As características dos frutos são importantes para a comercialização do café(VACARELLI \& MEDINA FILHO, 2003) e estão relacionadas com o rendimento da colheita. Para porcentagem de frutos chochos (Tabela 3), com exceção da cultivar 'Topázio’, os cafeeiros enxertados não apresentaram problemas de fecundação e formação da semente, corroborando os resultados de BERTRAND et al. (2001), os quais observaram que os porta-enxertos Coffea canephora e Coffea liberica não afetaram a quantidade de frutos chochos, grãos moca e teores de cafeína, lipídeos e sacarose dos cafeeiros Coffea arabica.

Ciência Rural, v.42, n.7, jul, 2012. 
Tabela 2 - Valores médios de produtividade para a interação de tipos de enxertia (mudas Enxertadas = Enx; Auto-enxertadas = A-Enx. e PéFranco $=$ P.F.) dentro de cultivares em cada colheita estudada.

\begin{tabular}{|c|c|c|c|c|c|c|}
\hline Cultivares & Tipos de enxertia & 2007 & 2008 & 2009 & 2010 & Médias \\
\hline \multirow{3}{*}{ ‘Acauã' } & Enx. & $70,6 \mathrm{bB}$ & $44,8 \mathrm{aC}$ & $6,2 \mathrm{cD}$ & $116,2 \mathrm{aA}$ & $59,5 \mathrm{a}$ \\
\hline & A-Enx. & $103,0 \mathrm{aA}$ & $54,8 \mathrm{aB}$ & $12,6 \mathrm{bC}$ & 99,7 aA & 67,5 a \\
\hline & P.F. & $111,7 \mathrm{aA}$ & 31,2 bC & $29,2 \mathrm{aC}$ & $76,7 \mathrm{bB}$ & $62,2 \mathrm{a}$ \\
\hline \multirow{3}{*}{ ‘Catucaí' } & Enx. & 84,3 aA & $27,1 \mathrm{aB}$ & $8,1 \mathrm{cB}$ & 75,3 aA & 48,7 a \\
\hline & A-Enx & 98,9 aA & 13,7 bC & $10,5 \mathrm{bC}$ & $73,7 \mathrm{aB}$ & 49,2 a \\
\hline & P.F. & $108,2 \mathrm{aA}$ & $1,9 \mathrm{bC}$ & $13,7 \mathrm{aC}$ & $64,9 \mathrm{aB}$ & 47,2 a \\
\hline \multirow{3}{*}{ 'Obatã' } & Enx. & $58,8 \mathrm{bA}$ & $15,9 \mathrm{aB}$ & $20,1 \mathrm{cB}$ & $60,1 \mathrm{aA}$ & 38,8 a \\
\hline & A-Enx & 98,9 aA & $3,0 \mathrm{aD}$ & $27,5 \mathrm{aC}$ & $57,1 \mathrm{aB}$ & 46,6 a \\
\hline & P.F. & $100,1 \mathrm{aA}$ & $2,5 \mathrm{aC}$ & $22,7 \mathrm{bC}$ & $61,7 \mathrm{aB}$ & 46,8 a \\
\hline \multirow{3}{*}{ 'Oeiras’ } & Enx. & $72,7 \mathrm{aA}$ & $39,2 \mathrm{aB}$ & $2,0 \mathrm{cC}$ & 80,1 aA & 48,5 a \\
\hline & A-Enx & $95,4 \mathrm{aA}$ & $37,6 \mathrm{aC}$ & $18,1 \mathrm{aC}$ & $65,5 \mathrm{aB}$ & 54,2 a \\
\hline & P.F. & 97,8 aA & $25,8 \mathrm{aC}$ & $14,2 \mathrm{bC}$ & $67,9 \mathrm{aB}$ & 51,4 a \\
\hline \multirow{3}{*}{ 'Palma II' } & Enx. & 83,3 aB & $60,7 \mathrm{aC}$ & $2,9 \mathrm{cD}$ & $120,0 \mathrm{aA}$ & 66,7 a \\
\hline & A-Enx & $101,2 \mathrm{aA}$ & $24,4 \mathrm{bB}$ & 19,6 bB & $104,9 \mathrm{aA}$ & $62,6 \mathrm{a}$ \\
\hline & P.F. & $86,2 \mathrm{aA}$ & $7,0 \mathrm{cB}$ & $27,2 \mathrm{aB}$ & $84,0 \mathrm{bA}$ & $51,1 \mathrm{~b}$ \\
\hline \multirow{3}{*}{ ‘Paraíso’ } & Enx. & $38,0 \mathrm{bB}$ & $40,7 \mathrm{aB}$ & $1,2 \mathrm{cC}$ & $77,8 \mathrm{aA}$ & $39,4 \mathrm{~b}$ \\
\hline & A-Enx & 87,9 aA & $25,8 \mathrm{bB}$ & 8,6 bB & 90,9 aA & 53,3 a \\
\hline & P.F. & $85,6 \mathrm{aA}$ & $14,3 \mathrm{bB}$ & $24,9 \mathrm{aB}$ & $75,6 \mathrm{aA}$ & 50,1 a \\
\hline \multirow{3}{*}{ ‘Topázio’’ } & Enx. & $90,1 \mathrm{aA}$ & $25,0 \mathrm{aB}$ & $6,2 \mathrm{bB}$ & $85,0 \mathrm{aA}$ & $51,6 \mathrm{a}$ \\
\hline & A-Enx & 87,3 aA & $31,7 \mathrm{aB}$ & $14,8 \mathrm{aB}$ & $67,2 \mathrm{aA}$ & 50,3 a \\
\hline & P.F. & $98,3 \mathrm{aA}$ & $17,7 \mathrm{aC}$ & $16,6 \mathrm{Ac}$ & $67,4 \mathrm{aB}$ & $50,0 \mathrm{a}$ \\
\hline \multicolumn{2}{|c|}{ Média Enxertado } & $71,1 \mathrm{bB}$ & $36,2 \mathrm{aC}$ & $6,7 \mathrm{cD}$ & 87,8 aA & 50,5 a \\
\hline \multicolumn{2}{|c|}{ Media Auto-Enxertado } & 96,1 aA & $27,3 \mathrm{bC}$ & $15,9 \mathrm{bD}$ & $79,8 \mathrm{bB}$ & 54,8 a \\
\hline \multicolumn{2}{|c|}{ Media Pé-Franco } & $98,2 \mathrm{aA}$ & $14,4 \mathrm{cC}$ & $21,2 \mathrm{aC}$ & $71,2 \mathrm{bB}$ & 51,3 a \\
\hline
\end{tabular}

Dentro de cada cultivar, médias seguidas de mesma letra minúscula nas colunas e maiúscula nas linhas não diferem significativamente entre si pelo teste de Scott-Knott a 5\% de probabilidade.

Em relação à porcentagem de grãos peneira 17 acima, apenas os cafeeiros enxertados das cultivares 'IBC Palma II’ e ‘Paraíso MG H 419-1’ tiveram redução neste percentual em relação aos auto enxertados e de pé franco. De maneira semelhante, BERTRAND et al. (2001), trabalhando as cultivares 'Caturra' e 'Catimor T5175' enxertadas em Coffea canephora e Coffea liberica, observaram que o uso destes porta-enxertos causaram redução do tamanho dos grãos e do aroma do produto final.

Ao avaliar as características anatômicas do caule de cafeeiros, DIAS et al. (2009b) concluíram que a enxertia de $\boldsymbol{C}$. arabica sobre 'Apoatã IAC 2258' não apresenta sinais evidentes de incompatibilidade de reconstituição e formação de tecidos. No presente trabalho, observa-se que, com exceção de 'IBC Palma II' e 'Paraíso MG H 419-1', as cultivares estudadas apresentaram comportamento agronômico satisfatório em todas as características avaliadas e semelhante ao pé-franco.

Para a característica uniformidade de maturação, nota-se significante variação de resultados para as cultivares dentro dos grupos de plantas oriundas de mudas enxertadas e auto enxertadas. 
Tabela 3 - Valores médios de porcentagem de frutos chochos (\% FC), porcentagem de grãos peneira 17 acima (\% P17), porcentagem de frutos verdes (\% FV), porcentagem de frutos cereja (\% FCE) e porcentagem de frutos passa/secos (\% FP/S) das colheitas 2008 a 2010 para as diferentes interações de tipos de enxertia x cultivares.

\begin{tabular}{|c|c|c|c|c|c|c|}
\hline Cultivares & Tipos de enxertia & $\% \mathrm{FC}$ & $\%$ P17 & $\% \mathrm{FV}$ & $\%$ FCE & $\% \mathrm{FP} / \mathrm{S}$ \\
\hline \multirow{3}{*}{ ‘Acauã’ } & Enx. & 15,9 a & 31,0 a & $18,0 \mathrm{a}$ & 52,5 a & 29,4 a \\
\hline & A.-Enx. & 11,5 a & 34,9 a & 20,2 a & 54,7 a & 25,2 a \\
\hline & Pé-Franco & 11,8 a & 38,5 a & $19,1 \mathrm{a}$ & 56,1 a & 24,8 a \\
\hline \multirow{3}{*}{ ‘Catucaí’ } & Enx. & 15,5 a & 50,6 a & 14,9 a & 57,3 a & 27,9 a \\
\hline & A.-Enx. & 13,8 a & 56,9 a & $13,4 \mathrm{a}$ & 60,7 a & 25,8 a \\
\hline & Pé-Franco & 13,3 a & 50,8 a & $17,2 \mathrm{a}$ & 56,7 a & 26,0 a \\
\hline \multirow{3}{*}{ ‘Obatã’ } & Enx. & 11,3 a & 57,0 a & $12,0 \mathrm{a}$ & 61,6 a & 26,4 a \\
\hline & A.-Enx. & 10,2 a & 59,9 a & 20,4 a & 53,4 a & 26,1 a \\
\hline & Pé-Franco & 11,3 a & 57,4 a & $19,1 \mathrm{a}$ & 59,4 a & $21,4 \mathrm{a}$ \\
\hline \multirow{3}{*}{ ‘Oeiras’ } & Enx. & 7,9 a & 42,4 a & $10,5 \mathrm{a}$ & 55,3 a & 34,1 a \\
\hline & A.-Enx. & 10,5 a & 46,8 a & 15,2 a & 53,3 a & 31,4 a \\
\hline & Pé-Franco & 9,6 a & $41,1 \mathrm{a}$ & $11,2 \mathrm{a}$ & 56,1 a & 32,6 a \\
\hline \multirow{3}{*}{ ‘Palma II’ } & Enx. & 8,1 a & $48,7 \mathrm{~b}$ & 20,5 a & $54,0 \mathrm{~b}$ & $25,5 \mathrm{~b}$ \\
\hline & A.-Enx. & 11,7 a & 61,2 a & $38,6 \mathrm{~b}$ & $49,0 \mathrm{~b}$ & 12,4 a \\
\hline & Pé-Franco & $10,0 \mathrm{a}$ & 62,5 a & 26,3 b & $60,4 \mathrm{a}$ & 13,3 a \\
\hline \multirow{3}{*}{ ‘Paraíso’ } & Enx. & $17,5 \mathrm{a}$ & $28,0 \mathrm{~b}$ & $22,0 \mathrm{a}$ & $60,1 \mathrm{a}$ & 17,8 a \\
\hline & A.-Enx. & 15,5 a & 40,8 a & $29,1 \mathrm{~b}$ & 59,2 a & 11,7 a \\
\hline & Pé-Franco & 24,3 a & 36,3 a & $33,6 \mathrm{~b}$ & $50,3 \mathrm{~b}$ & 16,0 a \\
\hline \multirow{3}{*}{ ‘Topázio’ } & Enx. & $12,3 \mathrm{a}$ & 42,8 a & $11,2 \mathrm{a}$ & $64,2 \mathrm{a}$ & 24,5 a \\
\hline & A.-Enx. & $31,7 \mathrm{~b}$ & 40,3 a & $15,4 \mathrm{a}$ & 59,9 a & 24,5 a \\
\hline & Pé-Franco & 18,3 a & 44,0 a & 15,3 a & 59,7 a & $24,8 \mathrm{a}$ \\
\hline
\end{tabular}

Dentro de cada cultivar, médias seguidas de mesma letra minúscula nas colunas não diferem significativamente entre si pelo teste de ScottKnott a 5\% de probabilidade.

Verificou-se que a cultivar 'IBC Palma II' foi influenciada pela enxertia, mostrando-se de maturação precoce ao apresentar maior porcentagem de frutos passa/secos. Esta mesma cultivar, quando de pé franco e auto enxertada, apresentou maturação dos frutos mais tardia, observada pela maior porcentagem de frutos verdes. A cultivar 'Paraíso MG H 419-1' quando em pé-franco e auto-enxertada mostrou-se de maturação mais tardia que as plantas enxertadas. As demais cultivares não apresentaram diferenças significativas entre os três tipos de enxertia utilizados.

\section{CONCLUSÃO}

As cultivares enxertadas estudadas apresentaram comportamento agronômico satisfatório em todas as características avaliadas e semelhante ao pé-franco. Em áreas isentas de fitonematoides, à exceção das cultivares ‘IBC Palma II’ e 'Paraíso MG H 419-1', as demais cultivares estudadas são adequadas opções de escolha para uso como copas. A técnica da enxertia com o uso do porta-enxerto ‘Apoatã IAC 2258’ em área isenta de fitonematoides não se justifica para incremento de produtividade.

\section{AGRADECIMENTOS}

Os autores agradecem à Fundação de Amparo à Pesquisa do Estado de Minas Gerais (FAPEMIG), ao Consórcio Brasileiro de Pesquisas Cafeeiras e ao Instituto Nacional de Ciência e Tecnologia do Café (INCT Café), pelo financiamento do projeto, e ao Conselho Nacional de Desenvolvimento Científico e Tecnológico (CNPq), pela concessão da bolsa de estudos.

\section{REFERÊNCIAS}

ALFONSI, E.L. et al. Crescimento, fotossíntese e composição mineral em genótipos de Coffea com potencial para utilização como 
porta-enxerto. Bragantia, v.64, n.1, p. 1-13, 2005. Disponível em: $<$ http://www.scielo.br/scielo.php?script=sci_arttext\&pid=S000687052011000100006\&lng=pt\&nrm=iso>. Acesso em: 25 fev. 2011. doi: 10.1590/S0006-87052011000100006.

ANTUNES FILHO H.; CARVALHO, A. Melhoramento do cafeeiro, ocorrência de lojas vazias em frutos de café Mundo Novo. Bragantia, v.13, p.165-179, 1954. Disponível em: <http://www.iac.sp.gov.br/bragantia/volume/1301/130114.pdf>. Acesso em: 21 jun. 2011. doi 10.1590/S0006-87052011000100006.

BERTRAND, B. et al. Growth, production, and bean quality of Coffea arabica as a interspecific grafting: consequences for rootstock breeding. HortScience, v.36, n.2, p.269-273, 2001. Disponível em: <http://publications.cirad.fr/une_notice.php?dk=525515>. Acesso em: 14 jun. 2011.

BRASIL. Ministério da Agricultura, pecuária e Abastecimento. Instrução Normativa n.8, 11 de junho de 2003. Disponível em: $<$ http:www.abic.com.br/arquivos/abic_nm_a1d_inst_normativa08.pdf > Acesso em: 03 jul. 2011.

CABOS, R.Y.M. et al. Evaluation of coffee genotypes for rootknot nematode resistance. Nematropica v.40, n.2, p.191-202, 2010. Disponível em: <http://abis.upc.es/bionta/sites/default/files/ 2-17551_p191-202_nemdj.pdf>. Acesso em: 14 jun. 2011.

CARVALHO S.P. Metodologias de avaliação do desempenho de progênies do cafeeiro (Coffea arabica L.). 1989. 68f. Dissertação (Mestrado em Genética e Melhoramento de Plantas) - Universidade Federal de Lavras, MG.

DIAS F.P. et al. Caracterização de progênies do cafeeiro (Coffea arabica L.) selecionadas em Minas Gerais: II - Caracteres relacionados à produção. Revista Ceres, v.52, p.85-100, 2005. Disponível em: <http://www.ceres.ufv.br/CERES/revistas/ V52N299P00705.pdf>. Acesso em: 04 jul. 2011.

DIAS, F.P. et al. Produção de cafeeiros Coffea arabica L. pés francos autoenxertados e enxertados em Apoatã IAC 2258. Ciência e Agrotecnologia, v.33, n.2, p. 484-487, 2009a. Disponível em: $<$ http://www.scielo.br/scielo.php?script=sci_arttext\&pid=S141370542009000200019\&lng=en\&nrm=iso>. Acesso em: 04 jul. 2011.

DIAS, F.P. et al. Estudo anatômico de cafeeiros enxertados. Ciência e Agrotecnologia, v.33, n.3, p.735-742, 2009b. Disponível em: $<$ http://www.scielo.br/scielo.php?script=sci_arttext\&pid=S141370542009000300011\&lng=en\&nrm=iso $>$. Acesso em: 25 fev. 2011. doi: 10.1590/S1413-70542009000300011.

FAHL, J.I. et al. Enxertia de Coffea arabica sobre progênies de $\boldsymbol{C}$. canephora e de $\boldsymbol{C}$. congensis no crescimento, nutrição mineral e produção. Bragantia, v.57, n.2, p.297-312, 1998. Disponível em:
$<$ http://www.scielo.br/scielo.php?script=sci_arttext\&pid= S000687051998000200012\&lng=en\&nrm=iso>. Acesso em: 15 fev. 2011. doi: 10.1590/S0006-87051998000200011.

FAZUOLI, L.C. Avaliação de progênies de café “Mundo Novo” (Coffea arabica L.). 1977. 146f. Dissertação (Mestrado em Agronomia) - Escola Superior de Agricultura "Luiz de Queiroz”, Piracicaba, SP.

FERREIRA, D.F. SISVAR: um programa para análises e ensino de estatística. Revista Symposium, v.6, p.36-41, 2008. Disponível em: <http://www.fadminas.org.br/symposium/ 12_edicoes/artigo_5.pdf>. Acesso em: 10 ago. 2011.

FERREIRA, A.D. et al. Absorção, translocação e eficiência no uso dos macronutrientes em cafeeiros (Coffea arabica) enxertados em Apoatã IAC 2258 (Coffea canephora). Interciencia, v.35, p.1-5, 2010. Disponível em: <http:// www.interciencia.org/v35_11/818.pdf>. Acesso em: 10 ago. 2011.

PAIVA R.N. et al. Comportamento agronômico de progênies de cafeeiro (Coffea arabica L.) em Varginha-MG. Coffee Science, v.5, p.49-58, 2010. Disponível em: <http:// www.coffeescience.ufla.br/ojs/index.php/Coffeescience/article/ viewArticle/271>. Acesso em: 07 ago. 2011.

PEREIRA, S.P. et al. Crescimento, produtividade e bienalidade do cafeeiro em função do espaçamento de cultivo. Pesquisa Agropecuária Brasileira, v.46, n.2, p.152-160, 2011. Disponível em: <http://www.scielo.br/pdf/pab/v46n2/ v46n02a06.pdf>. Acesso em: 27 dez. 2011. doi: 10.1590/ S0100-204X2010001200010.

TOMAZ, M.A. et al. Porta-enxertos afetando o desenvolvimento de mudas de Coffea arabica L. Ciência Rural, v.35, n.6, p.570-575, 2005. Disponível em: <http:// www.scielo.br/scielo.php?script=sci_arttext\&pid=S0103$84782005000300012 \& \operatorname{lng}=e n \& n r m=i s o>$. Acesso em: 07 ago. 2011. doi: 10.1590/S0103-84782005000300011.

TOMAZ, M.A. et al. Diferenças genéticas na eficiência de absorção, na translocação e na utilização de $\mathrm{K}$, Ca e $\mathrm{Mg}$ em mudas enxertadas de cafeeiro. Ciência Rural, v.38, n.6, 15401546, 2008. Disponível em: <http://www.scielo.br/ s cielo.ph p ? s c ript = s ci_art tex t \& pid=S 0103 84782008000600008\&lng $=$ en $\& n r m=i s o>$. Acesso em: 14 jun. 2011. doi: 10.1590/S0103-84782008000600008.

VACARELLI, V.; MEDINA FILHO, H. Avaliação de frutos chochos e de sementes do tipo moca no rendimento de híbridos arabustas tetraplóides (Coffea arabica x C. canephora). Bioscience Journal, v.19, n.3, p. 155-165, 2003. Disponível em: <http://www.seer.ufu.br/index.php/biosciencejournal/issue/ view/388>. Acesso em: 07 ago. 2011. 